\section{Estimulação ambiental e uso do andador infantil por lactentes com desenvolvimento normal}

\section{Environmental stimulation and use of a children's baby walkers by infants with normal development}

Karolina Alves de Albuquerque 1

Marisa Cotta Mancini 2

Adriana de França Drummond 3

Luis Megale 4

Paula Silva de Carvalho Chagas 5

\section{Abstract}

Objectives: to evaluate the quantity and quality of environmental stimuli available to infants with normal development who use a baby walkers prior to learning to walk by themselves.

Methods: a cross-sectional study was carried out with 24 infants distributed into two groups of twelve: one group of children who had used a baby walkers $(B W)$ and 12 who had not $(C)$, with the children in both groups being of equivalent ages, sex, and socio-economic background. The Home Observation for Measurement of the Environment (HOME) test provided a documentary Record of the stimuli provided by the environment. Student's t-test for independent samples was used to compare the mean scores on the HOME test of both groups, considering the level of significance to be $\alpha=0.05$.

Results: differences in the HOME scores, with the $B W$ group obtaining higher scores than the control group ( $p=0.014)$, with a low magnitude effect $(d=0.24)$.

Conclusions: the use of a children's baby walkers in the period before learning to walk by infants with normal development may be associated with more stimulating home environments.

Key words Environment, Child development, Child

\section{Resumo}

Objetivos: avaliar a quantidade e qualidade de estímulos ambientais disponiveis para lactentes com desenvolvimento normal que fizeram uso do andador infantil anteriormente à aquisição da marcha independente.

Métodos: estudo transversal, com 24 lactentes distribuidos em dois grupos, sendo 12 do grupo exposto ao andador infantil (AI) e 12 do grupo não-exposto $(C)$, mantendo-se equivalência entre grupos em idade, sexo e nível sócio econômico da familia. O teste Observation for Measurement of the Environment (HOME) documentou os estimulos oferecidos pelo ambiente. Teste-t de Student para amostras independentes comparou os escores médios do teste HOME de ambos os grupos, considerando o nivel de significância $\alpha=0,05$.

Resultados: diferenças no teste HOME foram observadas, tendo o grupo AI obtido escores superiores ao grupo $C(p=0,014)$, com efeito de magnitude fraca $(d=0,24)$.

Conclusões: o uso do voador infantil no periodo pré-aquisição da marcha por lactentes com desenvolvimento normal, pode estar associado a ambientes domiciliares mais estimuladores.

Palavras-chave Ambiente, Desenvolvimento infantil, Criança 


\section{Introdução}

Aquisições do desenvolvimento infantil emergem na medida em que a criança se envolve ativamente com os ambientes físico e social de seu cotidiano. $\mathrm{O}$ processo de desenvolvimento caracteriza-se por mudança permanente na forma como estas crianças percebem e lidam com o ambiente. ${ }^{1}$ Nessa perspectiva ecológica, o desenvolvimento infantil influencia e é influenciado ativamente pelas transações da criança com o meio em que vive. ${ }^{2}$

Os principais fatores de risco e de proteção para desfechos do desenvolvimento de lactentes estão presentes nos múltiplos contextos nos quais a criança está inserida, ilustrados pela família, escola e comunidade. 2 Atualmente, alguns autores argumentam que os estímulos disponíveis no ambiente podem influenciar diretamente desfechos positivos ou negativos do desenvolvimento infantil.3-5

Estudos demonstraram associação positiva entre a estimulação disponível no ambiente doméstico e o desempenho motor e mental de lactentes, sendo tal associação mediada pela estabilidade psicossocial da família.3,4 Dessa forma, os cuidados e estímulos oferecidos ao lactente pela família ilustram a qualidade do ambiente no qual ela vive, podendo ser determinantes no processo de aprendizagem e no desenvolvimento da criança, principalmente na primeira infância. 3,5

Diferenças nas práticas maternas podem impactar no desenvolvimento de lactentes, uma vez que a oportunidade para praticar habilidades emergentes é oferecida pelos pais no ambiente onde o lactente está inserido. Práticas maternas são comportamentos específicos direcionados a determinadas metas, através das quais a família cumpre as expectativas do papel de cuidador de referência. 6

Uma das estratégias utilizadas pelos pais para promover a emergência da marcha de lactentes é o uso do andador infantil, também denominado "voador" ou "anda já". Apesar da polêmica vigente acerca dos efeitos negativos atribuídos ao andador infantil, 7 alguns pais acreditam que o uso deste equipamento mantém a criança calma e entretida no ambiente domiciliar, podendo estimular a aquisição de marcha. ${ }^{8}$ Mesmo tendo o uso e distribuição deste equipamento sido proibidos em alguns países como o Canadá, no Brasil, as orientações negativas disponibilizadas por profissionais da saúde não têm necessariamente promovido efeito na opção das famílias por fazer uso do mesmo com seus filhos.

Diferenças nas práticas maternas ilustradas pela estimulação do ambiente domiciliar, bem como pela opção de usar o andador infantil com o próprio filho(a) podem influenciar o desenvolvimento de lactentes. Entretanto, até o momento, a relação entre uso do andador infantil e estimulação do ambiente doméstico permanece como hipótese ainda não testada empiricamente. O objetivo deste estudo foi avaliar a estimulação ambiental disponível para lactentes com desenvolvimento normal que estejam fazendo uso do andador infantil anteriormente à aquisição da marcha independente.

\section{Métodos}

Trata-se de estudo transversal para o qual foram selecionados por conveniência lactentes com desenvolvimento normal distribuídos em dois grupos, sendo um grupo exposto ao andador infantil $(\mathrm{AI}=$ andador infantil) e o outro grupo não-exposto ( $\mathrm{C}=$ controle). O cálculo amostral pautou-se em efeito moderado da variável resposta estimado entre 0,5 e 0,6 , poder estatístico 0,80 e nível de significância igual a $0,05.7$ Uma amostra com 12 participantes por grupo seria adequada para demonstrar o efeito de interesse.

O grupo AI incluiu lactentes cujos pais relataram fazer uso do andador infantil no período anterior à aquisição da marcha por escolha e iniciativa dos mesmos; o grupo $\mathrm{C}$ foi composto por lactentes que não usaram o andador, de faixa etária, sexo e nível socioeconômico equivalentes aos participantes do grupo AI. Características socioeconômicas foram classificadas pelo Critério de Classificação Econômica Brasil, da Associação Brasileira de Empresas de Pesquisa. ${ }^{9}$

A quantidade e qualidade dos estímulos oferecidos pelo ambiente foram avaliadas pela versão revisada do teste Home Observation for Measurement of the Environment (HOME), 10 um teste desenvolvido para medir a quantidade e qualidade de apoio social, emocional e cognitivo disponível para a criança em seu ambiente familiar. ${ }^{11}$ Neste estudo, foi usada a versão para o grupo etário entre 0 e 3 anos (Infant-Toddler HOME), composto por 45 itens. O Infant-Toddler HOME inclui seis subescalas: 1) responsividade emocional e verbal do cuidador; 2) ausência de punição e restrição; 3) organização do ambiente físico e temporal; 4) disponibilidade de materiais, brinquedos e jogos apropriados; 5) envolvimento do cuidador com a criança; e 6) oportunidade de variação na estimulação diária. ${ }^{10} \mathrm{Os}$ escores de cada subescala são obtidos pela soma de seus itens, e o escore total é resultante do somatório das subescalas.

O inventário HOME foi administrado entre setembro e dezembro de 2008 por entrevista com a 
mãe, pai ou cuidador, acrescida de observação da relação mãe/pai/cuidador e filho/criança. As crianças participantes eram provenientes da região metropolitana de Belo Horizonte, Estado de Minas Gerais, e arredores, segundo critérios citados anteriormente. A partir da identificação dos lactentes que estavam em uso ou não do andador infantil, as famílias receberam uma visita domiciliar em que os pais responderam a um questionário com informações sobre a criança, e sobre possíveis fatores que pudessem interferir no ambiente familiar.

As crianças de ambos os grupos foram avaliadas de forma independente por dois examinadores previamente treinados na aplicação do teste HOME. Após o treinamento, a consistência entre ambos foi testada obtendo-se índice elevado de concordância $(\mathrm{ICC}=0,98)$. Os responsáveis assinaram termo de consentimento livre e esclarecido e este estudo foi aprovado pelo Comitê de Ética em Pesquisa da Universidade Federal de Minas Gerais (parecer $n^{\circ}$ ETIC 321/08).

A análise descritiva inicial utilizou o teste-t de Student para avaliar a equivalência entre os grupos quanto a idade e número de pessoas residentes no domicílio, e o teste qui-quadrado foi utilizado para as variáveis sexo das crianças, nível socioeconômico da família, e grau de instrução da mãe.

Teste-t de Student para amostras independentes comparou as médias dos grupos $\mathrm{AI}$ e $\mathrm{C}$ nos escores totais e das subescalas. O tamanho de efeito (d) foi calculado para cada uma das análises inferenciais. As análises consideraram nível de significância $\alpha=0,05$ sendo realizadas pelo programa Statistical
Package for Social Sciences (SPSS ${ }^{\circledR}$ ), versão 15.0 (SPSS Inc., 2006).

\section{Resultados}

A amostra foi constituída por 24 lactentes entre sete e dez meses de idade pós-termo, sendo 12 do grupo AI e 12 do grupo C. Quanto à classe socioeconômica, $62,5 \%$ das famílias eram de classe alta (A1 e A2), sendo o restante formado por famílias de classes média alta $(29,2 \%)$ e baixa $(8,3 \%)(\mathrm{B} 1, \mathrm{~B} 2$, e $\mathrm{C} 1)$. A Tabela 1 apresenta informações descritivas dos dois grupos em relação à idade e sexo das crianças, grau de escolaridade da mãe e número de residentes no domicílio. Não houve diferença significativa entre os grupos em nenhuma destas variáveis. Nenhuma perda amostral foi registrada.

Quanto à quantidade e qualidade dos estímulos ambientais, os resultados demonstraram diferenças significativas entre os grupos, tendo o grupo AI obtido escores superiores no teste Infant-Toddler HOME, comparado ao grupo $\mathrm{C}(p=0,014)$. O tamanho do efeito (d) foi de 0,24 , ilustrando pequena magnitude.

Comparações entre grupos nas diferentes subescalas (Tabela 2) revelaram que o grupo AI obteve escores significativamente superiores aos do grupo C em duas subescalas, a saber: subescala 3 organização do ambiente físico e temporal $(\mathrm{d}=0,20$; $p=0,029$ ) e subescala 5 - envolvimento do cuidador com a criança $(\mathrm{d}=0,18 ; p=0,038)$.

Tabela 1

Análise descritiva dos lactentes nos grupos andador infantil e controle nas variáveis idade, sexo, nível socioeconômico da família, grau de escolaridade da mãe e número de residentes na casa.

\begin{tabular}{|c|c|c|c|}
\hline \multirow[t]{2}{*}{ Variáveis descritivas } & \multicolumn{2}{|c|}{ Grupos } & \multirow[t]{2}{*}{$p$} \\
\hline & Andador infantil & Controle & \\
\hline Idade, $\bar{x}(D P)$ & $8 m 29 d(21 d) *$ & $9 m 12 d(20 d) *$ & 0,673 \\
\hline \multicolumn{4}{|l|}{ Sexo } \\
\hline Feminino & 6 & 7 & 0,682 \\
\hline Masculino & 6 & 5 & \\
\hline \multicolumn{4}{|l|}{ Escolaridade da mãe } \\
\hline Superior completo & 8 & 9 & 0,879 \\
\hline Superior incompleto & 1 & 1 & \\
\hline $2^{\circ}$ grau completo & 3 & 2 & \\
\hline Residentes no domicílio, $\bar{X}(D P)$ & $3,58(0,66)$ & $3,25(0,86)$ & 0,581 \\
\hline
\end{tabular}

* $\mathrm{m}=$ meses; $\mathrm{d}=$ dias. 
Valores médios (DP) dos escores obtidos pelos grupos andador infantil e controle no teste Infant-Toddler Home e nas seis subescalas que compõem o teste.

\begin{tabular}{|c|c|c|c|c|c|}
\hline Variáveis dependentes & Grupos & $\bar{x} \pm D P$ & d & $\mathbf{t}$ & $p$ \\
\hline \multirow[t]{2}{*}{ Infant-Toddler Home } & $\mathrm{Al}$ & $39,33 \pm 2,49$ & 0,24 & 2,65 & 0,014 \\
\hline & C & $36,08 \pm 3,42$ & & & \\
\hline \multicolumn{6}{|l|}{ Subescala 1: respostas } \\
\hline \multirow[t]{2}{*}{ emocionais e verbais do cuidador } & Al & $9,92 \pm 0,79$ & 0,13 & 1,82 & 0,089 \\
\hline & $\mathrm{C}$ & $8,83 \pm 1,89$ & & & \\
\hline \multicolumn{6}{|l|}{ Subescala 2: ausência de } \\
\hline \multirow[t]{2}{*}{ punição e restrição } & $\mathrm{Al}$ & $7,08 \pm 0,79$ & 0,01 & 0,55 & 0,583 \\
\hline & $\mathrm{C}$ & $6,91 \pm 0,66$ & & & \\
\hline \multicolumn{6}{|l|}{ Subescala 3: organização do } \\
\hline \multirow[t]{2}{*}{ ambiente físico e temporal } & Al & $5,66 \pm 0,49$ & 0,2 & 2,33 & 0,029 \\
\hline & $\mathrm{C}$ & $4,91 \pm 0,99$ & & & \\
\hline \multicolumn{6}{|l|}{ Subescala 4: disponibilidade de } \\
\hline \multirow[t]{2}{*}{ materiais e brinquedos } & Al & $7,75 \pm 1,13$ & 0,1 & 1,52 & 0,141 \\
\hline & $\mathrm{C}$ & $7,08 \pm 0,99$ & & & \\
\hline \multicolumn{6}{|l|}{ Subescala 5: envolvimento do } \\
\hline \multirow[t]{2}{*}{ cuidador com a criança } & $\mathrm{Al}$ & $5,50 \pm 0,67$ & 0,18 & 2,20 & 0,038 \\
\hline & $\mathrm{C}$ & $4,75 \pm 0,96$ & & & \\
\hline \multicolumn{6}{|l|}{ Subescala 6: possibilidade de } \\
\hline \multirow[t]{2}{*}{ variação na estimulação diária } & Al & $3,41 \pm 0,99$ & 0,01 & $-0,43$ & 0,671 \\
\hline & $\mathrm{C}$ & $3,58 \pm 0,90$ & & & \\
\hline
\end{tabular}

$\mathrm{Al}=$ andador infantil; $\mathrm{C}=$ controle; $\mathrm{d}=$ tamanho do efeito $(\mathrm{Al}-\mathrm{C})$.

\section{Discussão}

Os resultados do presente estudo revelaram que lactentes do grupo AI parecem receber maior estimulação relacionada à organização do ambiente físico e temporal, e envolvimento do cuidador com a criança, comparados com lactentes do grupo C. Destaca-se que, até o momento, nenhum estudo havia avaliado a quantidade ou qualidade dos estímulos domiciliares disponibilizados pelos pais de lactentes que optaram por fazer uso do andador infantil. Considerando-se a relevância de se investigar as influências do ambiente no desenvolvimento infantil, visando contribuir para a compreensão da complexa rede de interações que influencia este processo de mudança, ${ }^{2}$ o presente estudo constitui investigação preliminar sobre o tema, devido ao reduzido tamanho amostral e ao desenho metodológico utilizado.

Os resultados sugerem um ambiente domiciliar mais enriquecido em qualidade de estímulos no grupo AI, mensurado pelo teste HOME, comparado com o do grupo C. Entretanto, considerando o pequeno tamanho deste efeito $(d=0,24)$, certamente o uso do andador infantil não resulta em diferença importante na estimulação domiciliar de lactentes com as características sociodemográficas deste grupo amostral. Na verdade, a estimulação do ambiente domiciliar parece constituir fenômeno de maior complexidade e, a partir dos resultados apresentados, futuras investigações poderão investigar este tema incluindo um espaço temporal entre a variável independente e desfecho.

No teste HOME, a organização ambiental referese à regularidade e previsibilidade dos cuidados oferecidos à criança na rotina familiar, e à utilização 
de serviços comunitários como parte do sistema de apoio. ${ }^{10} \mathrm{~A}$ diferença entre grupos nesta subescala sugere que familiares que usam o andador infantil parecem se preocupar mais com a segurança do ambiente físico e com a organização da rotina da criança. A subescala de envolvimento do cuidador com a criança informa sobre frequência e estabilidade do contato criança-cuidador, podendo-se inferir sobre o envolvimento ativo dos pais na aprendizagem da criança. 10 Por outro lado, não foram observadas diferenças significativas entre grupos nas demais subescalas do teste HOME, a saber, responsividade emocional/verbal do cuidador, ausência de punição/restrição, disponibilidade de materiais, brinquedos e jogos apropriados, e oportunidade de variação na estimulação diária. É possível que uma caracterização socioeconômica de ambos os grupos, que incluiu famílias de níveis mais elevados, possa justificar em parte, esta ausência efeitos significativos nestas subescalas.

\section{Referências}

1. Bronfenbrenner UA Ecologia do desenvolvimento humano: experimentos naturais e planejados. Porto Alegre: Artes Médicas; 1996.

2. Sameroff AJ. Transactional regulation and early intervention. In: Meisels SJ, Shonkoff JP. Handbook of early childhood intervention. Cambridge: Cambridge University Press; 1990. p. 119-49.

3. Guerra MQ. Fatores associados à qualidade da estimulação domiciliar de crianças aos 12 meses de idade da zona da mata meridional de Pernambuco [dissertação]. Recife: Universidade Federal de Pernambuco; 2002.

4. Andrade AS, Santos DN, Bastos AC, Pedromônico MRM, Almeida-Filho N, Barreto ML. Ambiente familiar e desenvolvimento cognitivo infantil: uma abordagem epidemiológica. Rev Saúde Pública. 2005; 39: 606-11.

5. Eickmann SH, Lira PI, Lima MC. Desenvolvimento menta e motor aos vinte e quatro meses de crianças nascidas a termo com baixo peso. Arq Neuropsiquiatr. 2002; 60: 74854

Recebido em 18 de agosto de 2010

Versão final apresentada em 21 de fevereiro de 2011

Aprovado em 24 de março de 2011
Como limitação desse estudo destaca-se o elevado nível socioeconômico das famílias participantes, que pode restringir a generalização dos resultados para famílias de níveis mais baixos. Em acréscimo, seleção amostral não aleatória pode não ter produzido grupos com características representativas da população de referência.

Os resultados deste estudo sugerem que o ambiente domiciliar de crianças que fizeram uso do andador infantil no período anterior à aquisição da marcha, parece disponibilizar maior qualidade de estímulos, comparados aos de lactentes que não utilizaram este equipamento. Estudos futuros poderão investigar se os efeitos dessa estimulação diferenciada do ambiente domiciliar apresentam maior magnitude em famílias de diferentes níveis socioeconômicos e se tais diferenças estão associadas a futuros desfechos no desenvolvimento infantil.

6. Darling N, Steinberg L. Parenting style as context: an integrative model. Psychol Bull. 1993; 113: 487-96.

7. Chagas PSC, Mancini MC. Efeitos do uso do andador infantil na aquisição da marcha em lactentes com desenvolvimento normal [tese]. Belo Horizonte: Universidade Federal de Minas Gerais; 2010.

8. DiLillo D, Damashek A, Peterson L. Maternal use of baby walkers with young children: recent trends and possible alternatives. Inj Prev. 2001; 7:223-7.

9. ABEP (Associação Brasileira de Empresas de Pesquisa). Critério de classificação econômica Brasil 2008. [acesso em 26 mai 2006]. Disponível em: www.abep.org

10. Caldwell BM, Bradley RH. Home inventory administration manual. Little Rock: University of Arkansas; 2003.

11. Totsika V, Sylva K. The Home Observation for Measurement of the Environment Revisited. Child Adolesc Ment Health. 2004; 9: 25-35. 